\title{
Geriatric Consultation Reduces High-risk Medication Usage at Discharge in Elderly Trauma Patients
}

Jyoti Sharma ${ }^{1}$, Manisha Parulekar ${ }^{2}$, Peter Stewart ${ }^{1}$, Melissa Blatt ${ }^{1}$, Tania Zielonka ${ }^{1}$, Themba Nyirenda ${ }^{3}$ , Christopher Rogers ${ }^{4}$, Lisa Tank ${ }^{4}$

1. Surgery, Hackensack University Medical Center, Hackensack, USA 2. Internal Medicine, Hackensack Univeristy Medical Center, Hackensack, USA 3. Miscellaneous, Hackensack University Medical Center, Hackensack, USA 4. Internal Medicine, Hackensack University Medical Center, Hackensack, USA

Corresponding author: Jyoti Sharma, jyoti.sharma@hackensackmeridian.org

\section{Abstract \\ Background}

Traumatic injury in a growing geriatric population is associated with higher mortality and complication rates. Geriatric consultation $(\mathrm{GC})$ is vital in reducing risk factors that contribute to adverse outcomes. This study aims to determine if receiving a GC had an impact on high-risk medication usage.

\section{Methods}

Patients eligible for a GC, age $\geqslant 65$, and length of stay > two days, were identified via a chart review from July 2013 to July 2014 at a Level II trauma center. This population was divided into those with and without a GC. Data collected included demographics, injury severity, medications, delirium, mortality, and readmissions. High-risk medications were defined using the Beers Criteria. Statistical analysis involved using appropriate standard tests to compare groups, including multivariate logistic regression.

\section{Results}

Forty-nine of a total of 104 patients received a GC. Groups were comparable on injury severity score, comorbidities, and high-risk medication use upon admissions. The GC group was $74 \%$ less likely to be discharged on high-risk medications than the non-GC group.

\section{Conclusion}

GC in elderly trauma patients reduces high-risk medication use upon discharge. Further studies are needed to explore how GC impacts readmission rates and mortality. A multidisciplinary trauma team, including a geriatrician, must exist to address the unique medical, psychological, functional, and social issues of a

Received 10/19/2018

Review began 11/14/2018

Review ended 11/22/2018

Published 11/28/2018

๑) Copyright 2018

Sharma et al. This is an open access

article distributed under the terms of the Creative Commons Attribution License CC-BY 3.0., which permits unrestricted use, distribution, and reproduction in any medium, provided the original author and source are credited.

Categories: General Surgery

Keywords: delirium, geriatric trauma, geriatric consultation, high risk medications

\section{Introduction}

In 2014, 46 million (15.0\%) or about one in every seven individuals were age 65 and older; however, by 2060 , this is expected to more than double to 98 million (1). Moreover, persons age 85 and older are estimated to grow significantly from 6 million in 2014 to 14.6 million in 2040 [1]. With a growing geriatric population, the incidence of traumatic injury will also increase [2]. As of 2013, unintentional injury was the eighth leading cause of death in the 65 and older population [3]. One in three older adults falls each year, leading to direct medical costs totaling $\$ 34$ billion in 2013 [4]. This cost, along with the total number of falls, is projected to increase significantly as the United States population continues to age [4].

High-risk medications, such as benzodiazepines, sedatives, and psychotropic drugs in elderly patients, have been associated with an increased risk of falls and delirium [5]. In a systematic review and meta-analysis, Leipzig, Cumming, and Tinetti [6] found that elderly patients who were prescribed psychotropic,

benzodiazepine, or sedative medications had higher odds of falling. The use of benzodiazepines, both in the inpatient as well as the outpatient setting, has been associated with adverse outcomes in the elderly such as increased sedation, decreased attention, anterograde amnesia, falls with associated fractures, hemorrhage with associated hypotension, hypoglycemic encephalopathy, and liver failure [7-11].

Trauma injury in the elderly is also associated with higher mortality and complication rates as compared to younger patients [2]. The management of an older population requires a multidisciplinary approach that 
takes into consideration the decreased physical reserve and the presence of multiple comorbidities in these patients [2]. Multiple studies have shown that a proactive geriatric consultation (GC) alone or within a formal geriatric protocol has been linked with fewer episodes of delirium, fewer in-hospital falls, less likelihood of discharge to a long-term care facility, and a shorter length of stay [2,5,12-14]. According to 22 randomized trials with greater than 10,000 subjects, those who received a GC followed by appropriate medical care were $25 \%$ more likely to be alive and in their own home at one year after discharge $[2,12-13]$.

A geriatrician's participation in medication reconciliation, pain management, disposition decisions, and advance care planning effectively reduces in-hospital complications in older individuals [12-13]. Few studies have examined the effect of GC on the use of polypharmacy at discharge. This study aims to determine if receiving a GC had an impact on high-risk medication use at discharge in elderly trauma patients.

\section{Materials And Methods}

A retrospective study was performed at Hackensack University Medical Center (HUMC), which is a level II trauma center with 775 beds and approximately 900 traumas per year. The institutional trauma registry was queried for all subjects age 65 and older from July 2013 to July 2014. Variables collected from the chart included demographics (age, gender, height, weight, body mass index (BMI), race, ethnicity), level of activation, mechanism of injury, admission GCS, injury severity score (ISS), co-morbidities, initial vital signs, the presence of delirium, hospital medications, the presence of a GC, and high-risk medication use (i.e., benzodiazepines, opiates, and sedatives). Primary outcomes included the length of stay (LOS), discharge medications, discharge locations, readmission rates, and in-hospital mortality. Composite variables were created and included the following: depression (i.e., the patient had a diagnosis of depression and/or was using medications for depression) and multi-traumatic brain injury (TBI) (one or more anatomical region of hemorrhage as indicated by computed tomography (CT) of the head).

The HUMC department of trauma has developed a protocol based on American College of Surgeons (ACS) Trauma Quality Improvement Program (TQIP) Geriatric Trauma Management Guidelines [2] to determine if geriatricians should be consulted at admission (Figure 1). This protocol highlighted trauma patient criteria that should prompt a GC, but the ultimate decision was left to the discretion of the trauma surgeon. Patients were excluded if they did not meet the criteria for a GC, if expected LOS was less than 48 hours, and if patients were in a moribund condition or died in the trauma bay. Geriatricians, in conjunction with the trauma team, provided a multidisciplinary patient-centered approach with a specific focus on pharmacokinetic, social, physiological, behavioral, and somatic health factors.

1. All patients $>75$ years of age, admitted to the trauma service

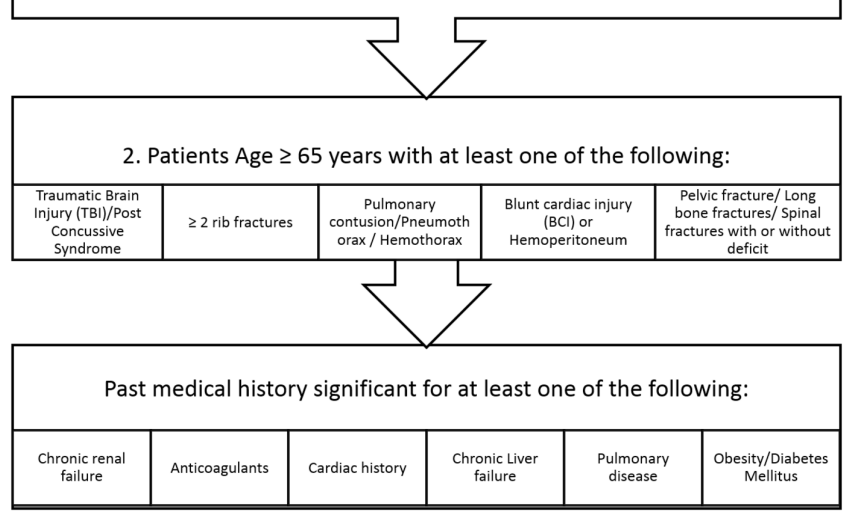

FIGURE 1: Criteria for Requesting a Geriatric Consult on Trauma Patients

Delirium was defined using the five key features from the Diagnostic and Statistical Manual of Mental Disorders, 5th Edition (DSM-V) [15], which include a disturbance: (1) in attention and awareness, (2) that develops over a short time period and is a shift from patient's baseline and fluctuates throughout the day, (3) in cognition, (4) that cannot be attributed to other previously existing conditions or disorders, and (5) cannot be accounted for by a medical condition, substance abuse/withdrawal, or medication side effect. Furthermore, for this retrospective analysis, the key components of delirium were identified via documentation of the following in the electronic medical record (EMR): positive confusion assessment method (CAM) scores [16], use of restraints/mitts, sitter, and confusion/agitation in the chart or delirium noted in the patient's problem list. 
In this study, high-risk medications were defined using the Beers criteria [17]. The Beers criteria (2) provides guidelines for safe and effective medication use while minimizing adverse drug events for the geriatric population. The criteria itself is a comprehensive medication list published elsewhere [17]. The variable "high-risk geriatric meds" included the following five categories of medications: anti-depressants, antipsychotics, benzodiazepines, sedatives, and opioids. Polypharmacy was defined as the use of more than five medications of any type at admission or discharge.

Descriptive analyses were conducted as follows: categorical/discrete variables were summarized as frequency (\%), continuous variables were reported as mean \pm standard deviation (SD) or median with interquartile range (IQR: 25 th - 75th percentile), depending on whether the data were normally distributed. The assumption of the normality of the data distribution was assessed using the Shapiro-Wilk test. A comparison of categorical variables between groups was conducted using the Pearson chi-square or Fisher's exact test, as appropriate. A comparison of continuous variables between groups was performed using a two-sided t-test for normally distributed data or Wilcoxon rank sum test for non-normally distributed data. Further, for a comparison of continuous variables between more than two groups, one-way analysis of variance (ANOVA) or the Kruskal-Wallis test, as appropriate, was conducted.

Univariate logistic regression analyses were performed to identify associations between variables. All of the variables that were significantly associated with being discharged on high-risk medications were entered in a multivariable model in which the stepwise selection procedure was employed to obtain a final model fit. Modeled covariates in univariate and multivariate logistic regression analyses were reported as odds ratio (OR) with $95 \%$ confidence intervals (CI). A p-value < 0.05 was chosen as the cut-off level for statistical significance. All data analysis was performed using SAS ${ }^{\mathrm{TM}}$ (version 9.4; SAS Institute Inc., Cary, NC, US).

Approval for the study was obtained from the HUMC institutional review board.

\section{Results}

A total of 104 charts were reviewed and met the criteria for geriatric consultation. Of these, 49 patients received a GC and 55 did not. Table 1 compares the demographic variables of both groups. Patients who received a GC were older (81.0 vs. 83.0 year old, $\mathrm{p}=0.041)$ and had a lower BMI (24.6 vs. $27.3 \mathrm{~kg} / \mathrm{m} 2, \mathrm{p}=$ $0.017)$ compared to those without. The GC group did appear to have slightly worse injuries in regards to injury severity score ( 9 vs. 10) although this finding was not statistically significant $(\mathrm{p}=0.052)$. Approximately70\% of patients in both groups had three or more co-morbidities. However, patients in the non-GC group were more likely to have pre-existing depression compared to those with GC (27.3\% vs. $10.2 \%$, $\mathrm{p}=0.046)$. Patients with a GC were more likely to be on an anticoagulant than those without $(26.5 \%$ vs. $10.9 \%, \mathrm{p}=0.040$ ). Table 1 also highlights delirium in the non-GC and GC groups. Most patients had a diagnosis of delirium prior to a GC. Patients with a GC and delirium were more likely to have restraints ordered $(\mathrm{p}=0.026)$, documentation of a CAM positive score on the step-down unit $(\mathrm{p}=0.017)$, and sitter usage ( $\mathrm{p}=0.017$ ) as compared to the non-GC group.

\begin{tabular}{|c|c|c|c|}
\hline Variables & Non-GC (N=55) & $\mathrm{GC}(\mathrm{N}=49)$ & P-Value \\
\hline Age, Median $\left(25^{\text {th }}-75^{\text {th }}\right)$, Years & $81.1(70.0-87.0)$ & $83.0(77.0-90.0)$ & $0.041^{*}$ \\
\hline Gender Female, n (\%) & 34 (61.8) & $28(57.1)$ & 0.691 \\
\hline Race & & & 0.237 \\
\hline White & $49(89.0)$ & $43(87.8)$ & \\
\hline Black & $1(1.8)$ & $4(8.1)$ & \\
\hline Asian & $5(9.0)$ & $2(4.1)$ & \\
\hline Ethnicity & & & 0.185 \\
\hline Non-Hispanic & $52(94.5)$ & $42(85.7)$ & \\
\hline Hispanic & $3(5.5)$ & $7(14.3)$ & \\
\hline BMI, median $\left(25^{\text {th }}-75^{\text {th }}\right), \mathrm{kg} / \mathrm{m}^{2}$ & $27.3(24.3-30.6)$ & $24.6(21.4-28.9)$ & $0.017^{*}$ \\
\hline Trauma Activation, N (\%) & & & 0.592 \\
\hline Level I & $2(3.6)$ & $3(6.1)$ & \\
\hline Level II & 34 (61.8) & $25(51.0)$ & \\
\hline Consult & $19(34.6)$ & $15(42.9)$ & \\
\hline
\end{tabular}




\section{Cureus}

Mechanism of Injury, N (\%)

MVA

Fall

Admission GCS, Median $\left(25^{\text {th }}-75^{\text {th }}\right)$

ISS, Median $\left(25^{\text {th }}-75^{\text {th }}\right)$

SICU Admission, n (\%)

Intubation, n (\%)

Injury, n (\%)

TBI

Major Fracture

Minor Fracture

Comorbidities, n (\%)

CHF

CAD

Cardiac Arrhythmia

HTN

CVA

Diabetes Mellitus

Lung Disease/COPD/Asthma

Liver Disease

Kidney Disease

Depression

Dementia

Parkinson

Alcoholism

Psychosis

Co-Morbidities $\geq 3, \mathrm{n}$ (\%)

Anticoagulant Use, n (\%)

Delirium, n (\%)

Delirium, all

At Admission

Prior to GC

Delirium Identifiers, n (\%)

Restraints Ordered

Mitts Ordered

CAM Positive - Step Down Unit

CAM Positive - ICU **

Sitter Usage

Delirium on Problem List

Agitation
$11.0(20.0)$

$44.0(80.0)$

$15.0(15.0-15.0)$

$9.0(5.0-12.0)$

17 (30.9)

$6(10.9)$

$22(40.0)$

27 (49.1)

16 (29.6)

9 (16.4)

18 (32.7)

18 (32.7)

48 (87.3)

8 (14.6)

17 (30.9)

7 (12.7)

1 (1.8)

2 (3.6)

15 (27.3)

8 (14.6)

2 (3.6)

5 (9.1)

$0(0.0)$

40 (72.7)

6 (10.9)

24 (43.6)

10 (18.2)

N/A

7 (12.7)

7 (12.7)

11 (20.0)

4 (23.5)

5 (9.1)

15 (27.8)

16 (29.6)
6 (12.2)

43.0 (87.8)

$15.0(15.0-15.0)$

0.227

$10(8.0-14.0)$

0.052

22 (44.9)

0.160

5 (10.2)

1.000

23 (46.9)

0.554

26 (53.1)

0.700

17 (34.7)

0.674

7 (14.3)

0.769

$17(34.7) \quad 0.832$

$15(30.6) \quad 0.817$

$\begin{array}{ll}39(79.6) & 0.305\end{array}$

$4(8.2) \quad 0.369$

$14(28.6) \quad 0.795$

$8(16.3) \quad 0.602$

$1(2.0) \quad 1.000$

$2(4.1) \quad 1.000$

$5(10.2) \quad 0.046^{*}$

$12(24.5) \quad 0.199$

$\begin{array}{ll}4(8.2) & 0.417\end{array}$

$0(0.0) \quad 0.059$

$1(2.0) \quad 0.476$

39 (79.6) $\quad 0.414$

$13(26.5) \quad 0.040^{*}$

$28(57.1) \quad 0.169$

$13(26.5) \quad 0.306$

$24(50.0) \quad$ N/A

$15(30.6) \quad 0.026^{*}$

8 (18.4) $\quad 0.426$

$20(41.7) \quad 0.017^{*}$

8 (36.4) $\quad 0.494$

$13(27.1) \quad 0.017^{\star}$

$19(40.4) \quad 0.180$

$13(27.1) \quad 0.776$ 


\section{Cureus}

Code Status on Discharge, $\mathrm{n}(\%)$

0.104

$\begin{array}{llc}\text { Full code } & 26(76.5) & 21(58.3) \\ \text { DNR } & 6(17.7) & 14(38.9) \\ \text { DNR/DNI } & 2(5.9) & 1(2.8)\end{array}$

\section{TABLE 1: Demographic Characteristics of Patients With and Without a Geriatric Consultation ( $\mathrm{N}=104)$}

BMI, body mass index; MVA, Motor vehicle accident; GCS, Glasgow coma scale; ISS, Injury severity score; SICU, Surgical intensive critical care unit; TBI, Traumatic brain injury; CHF, Congestive heart disease; CAD, Coronary artery disease; HTN, Hypertension; CVA, Cardiovascular accident; GC, Geriatric consultation; CAM, Confusion assessment method; ICU, Intensive care unit; DNR, Do not resuscitate; DNI, Do not intubate

${ }^{*}$ Statistically significant, $p<0.05$,

${ }^{*}$ Values may not add up to the total $\mathrm{N}$ due to missing variables

The non-GC and GC groups had comparable LOS, in-hospital mortality, and 30-day readmission (Table 2). The patients who received a GC were more likely to have a urinary tract infection (UTI) compared to the non-GC patients $(20.4 \%$ vs. $5.5 \%, \mathrm{p}=0.035)$. More patients in the non-GC group were likely to be discharged to acute rehabilitation than the GC patients; however, this was not statistically significant.

\begin{tabular}{|c|c|c|c|}
\hline Variables & Non-GC (N=55) & GC $(\mathrm{N}=49)$ & $P$ - value \\
\hline LOS, Median $\left(25^{\text {th }}-75^{\text {th }}\right)$, Days & $5.0(4.0-8.0)$ & $5.0(3.0-10.0)$ & 0.908 \\
\hline ICU LOS, Median $\left(25^{\text {th }}-75^{\text {th }}\right)$ & $0.0(0.0-1.0)$ & $0.0(0.0-2.0)$ & 0.162 \\
\hline In-hospital Mortality, n (\%) & $3(5.5)$ & $5(10.4)$ & 0.468 \\
\hline \multicolumn{4}{|l|}{ Morbidity, n (\%) } \\
\hline Pneumonia & $5(9.1)$ & $3(6.1)$ & 0.720 \\
\hline UTI & $3(5.5)$ & $10(20.4)$ & $0.035^{\star}$ \\
\hline DVT & $4(7.3)$ & $2(4.1)$ & 0.682 \\
\hline Renal Failure & $1(1.8)$ & $3(6.1)$ & 0.341 \\
\hline Discharge Location, n (\%) & & & 0.178 \\
\hline Home & $10(18.2)$ & $14(28.6)$ & 0.248 \\
\hline SNF & $14(25.5)$ & $14(28.6)$ & 0.826 \\
\hline Acute Rehab & $27(49.1)$ & $16(32.7)$ & 0.112 \\
\hline 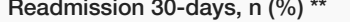 & $6(11.5)$ & $4(9.1)$ & 0.746 \\
\hline
\end{tabular}

\section{TABLE 2: Outcomes Variables in Elderly Trauma Patients With and Without GC}

LOS, Length of stay; ICU, Intensive care unit; UTI, Urinary tract infection; DVT, Deep vein thrombosis; SNF, Skilled nursing facility

* Statistically significant $p<0.05$

${ }^{*}$ Values may not add up to the total $\mathrm{N}$ due to missing variables

Table 3 highlights the high-risk medication status of geriatric trauma patients with and without a GC at three different time periods: at admission, in hospital, and at discharge. Both groups at admission had comparable uses of high-risk medications, but non-GC patients had a statistically significant increased usage of benzodiazepines and opioids. Despite the fact that patients in both the non-GC and the GC groups have a similar use of high-risk medications while in the hospital, the GC group was less likely to be 


\section{Cureus}

discharged on high-risk geriatric medications compared to the non-GC group $(73.1 \%$ vs. $47.7 \%, \mathrm{p}=$ $0.011)$. Lower rates of benzodiazepine and sedative use were also noted at discharge in the GC group $(26.9 \%$ vs. $4.4 \%, \mathrm{p}=0.003 ; 17.3 \%$ vs. $0.0 \%, \mathrm{p}=0.003$ ) versus the non-GC group.

\begin{tabular}{|c|c|c|c|}
\hline Variables & Non-GC (N=55) & GC (N=49) & P-value \\
\hline \multicolumn{4}{|l|}{ At Admission } \\
\hline \multicolumn{4}{|l|}{ Medication Type, n (\%) } \\
\hline Anti-Depressants & $17(30.9)$ & $15(30.6)$ & 0.974 \\
\hline Anti-Psychotics & $4(7.3)$ & $2(4.1)$ & 0.682 \\
\hline Benzodiazepines & $11(20.0)$ & $3(6.1)$ & $0.047^{*}$ \\
\hline Sedatives & $3(5.5)$ & $1(2.0)$ & 0.620 \\
\hline Opioids & $11(20.0)$ & $2(4.1)$ & $0.017^{\star}$ \\
\hline Polypharmacy (>5meds), n (\%) & $37(67.3)$ & $30(61.2)$ & 0.520 \\
\hline High-Risk Geriatric Meds, n (\%) & $29(52.7)$ & $21(42.9)$ & 0.315 \\
\hline \multicolumn{4}{|l|}{ In Hospital } \\
\hline \multicolumn{4}{|l|}{ Medication Type, n (\%) } \\
\hline Anti-Depressants & $15(27.3)$ & $14(28.6)$ & 0.883 \\
\hline Anti-Psychotics & $10(18.2)$ & $14(28.6)$ & 0.209 \\
\hline Benzodiazepines & $25(45.5)$ & $14(28.6)$ & 0.076 \\
\hline Sedatives & $16(29.1)$ & $14(28.6)$ & 0.954 \\
\hline Opioids & $42(76.4)$ & 39 (79.6) & 0.692 \\
\hline High-Risk Geriatric Meds, n (\%) & $50(90.9)$ & $45(91.8)$ & 1.000 \\
\hline \multicolumn{4}{|l|}{ At Discharge ** } \\
\hline \multicolumn{4}{|l|}{ Medication Iуре, n (\%) } \\
\hline Anti-Depressants & $15(28.9)$ & $12(26.7)$ & 0.811 \\
\hline Anti-Psychotics & $4(7.7)$ & $2(4.4)$ & 0.683 \\
\hline Benzodiazepines & $14(26.9)$ & $2(4.4)$ & $0.003^{\star}$ \\
\hline Sedatives & $9(17.3)$ & $0(0.0)$ & $0.003^{\star}$ \\
\hline Opioids & $26(50.0)$ & $15(33.3)$ & 0.096 \\
\hline Polypharmacy (>5meds), n (\%) & $44(84.6)$ & $42(95.5)$ & 0.103 \\
\hline High-Risk Geriatric Meds, n (\%) & 38 (73.1) & $21(47.7)$ & $0.011^{\star}$ \\
\hline
\end{tabular}

TABLE 3: Medication Status in Geriatric Trauma Patients With and Without a Geriatric Consultation

* Statistically significant $p<0.05$

${ }^{\star}$ Values may not add up to the total $\mathrm{N}$ due to missing variables

The univariate logistic analysis (Table 4) indicated that there was a statistically significant association between being discharged on high-risk medications and the following variables: BMI $(\mathrm{OR}=1.12)$, BMI overweight vs. normal $(\mathrm{OR}=3.83)$, BMI obese vs. normal ( $\mathrm{OR}=5.70)$, GC $(\mathrm{OR}=0.34)$, anti-depressant use on admission $(\mathrm{OR}=5.66)$, high-risk medication use on admission $(\mathrm{OR}=5.32)$, anti-depressant use during hospitalization $(\mathrm{OR}=26.47)$, depression $(\mathrm{OR}=6.06)$, multi-TBI $(\mathrm{OR}=0.23)$, subarachnoid hemorrhage $(\mathrm{OR}=$ $0.33)$, and the presence of a major fracture $(\mathrm{OR}=2.69)$. The final multivariable analysis model (Table 4$)$ 


\section{Cureus}

indicated that GC, depression, and multi-TBI were significantly and independently associated with being discharged on high-risk medications. The odds of being discharged on high-risk medications for patients who received GC were $74 \%$ lower than for patients who did not receive a GC (OR $=0.26,95 \%$ CI: 0.10 to 0.68 , $\mathrm{P}=0.006$ ). Having depression was associated with a seven-fold increased odds of being discharged on highrisk medications as compared to those not having depression ( $\mathrm{OR}=7.07,95 \% \mathrm{CI}: 2.02$ to $24.73, \mathrm{p}=0.002$ ). Patients with multi-TBI were also approximately $90 \%$ less likely to be discharged on high-risk medications than those without ( $\mathrm{OR}=0.11,95 \% \mathrm{CI}: 0.02$ to $0.65, \mathrm{p}=0.015)$. 


\section{Cureus}

\begin{tabular}{|c|c|c|c|c|c|c|}
\hline \multirow{2}{*}{ Variable } & \multicolumn{3}{|c|}{ Univariable } & \multicolumn{3}{|c|}{ Multivariable } \\
\hline & OR & $95 \% \mathrm{Cl}$ & $\mathrm{P}$ & OR & $95 \% \mathrm{Cl}$ & P-value \\
\hline BMI & 1.136 & $1.037-1.245$ & $0.006^{*}$ & & & \\
\hline BMI, Categories & & & $0.012^{x}$ & & & \\
\hline Underweight vs. Normal & 1.500 & $0.189-11.927$ & 0.702 & & & \\
\hline Overweight vs. Normal & 3.833 & $1.375-10.687$ & $0.010^{*}$ & & & \\
\hline Obese vs. Normal & 5.700 & $1.726-18.828$ & $0.004^{*}$ & & & \\
\hline GC vs. Non-GC & 0.336 & $0.144-0.788$ & $0.012^{x}$ & 0.255 & $0.096-0.680$ & $0.006^{\star}$ \\
\hline \multicolumn{7}{|l|}{ Medications at Admission } \\
\hline Anti-Depressants & 5.656 & $1.772-18.047$ & $0.003^{x}$ & & & \\
\hline Anti-Psychotics & 1.273 & $0.221-7.320$ & 0.787 & & & \\
\hline Benzodiazepines & 2.597 & $0.673-10.019$ & 0.166 & & & \\
\hline \multicolumn{7}{|l|}{ Sedatives } \\
\hline Opioids & 4.010 & $0.836-19.241$ & 0.083 & & & \\
\hline High-Risk Geriatric Meds & 5.232 & $2.090-13.101$ & $<0.001^{\star}$ & & & \\
\hline \multicolumn{7}{|l|}{ Medications During Hospitalization } \\
\hline Anti-Depressants & 26.470 & $3.397-206.236$ & $0.002^{x}$ & & & \\
\hline Anti-Psychotic & 1.333 & $0.482-3.691$ & 0.580 & & & \\
\hline Benzodiazepines & 2.127 & $0.874-5.174$ & 0.096 & & & \\
\hline Sedatives & 0.920 & $0.362-2.34$ & 0.862 & & & \\
\hline Upioids & 1.846 & $0.105-4.833$ & 0.212 & & & \\
\hline High-Risk Geriatric Meds & 13.526 & $1.590-115.048$ & $0.017^{\star}$ & & & \\
\hline Depression & 6.064 & $1.903-19.323$ & $0.002^{x}$ & 7.068 & $2.020-24.125$ & $0.002^{\star}$ \\
\hline \multicolumn{7}{|l|}{ Traumatic Injury } \\
\hline TBI & 0.524 & $0.227-1.208$ & 0.129 & & & \\
\hline Multt-IBI & 0.230 & $0.055-0.953$ & $0.043^{3}$ & 0.171 & $0.019-0.64 r$ & $0.015^{x}$ \\
\hline Subdural Hemorrhage & 0.735 & $0.291-1.856$ & 0.515 & & & \\
\hline Subarachnold Hemorrhage & 0.327 & $0.119-0.901$ & $0.031^{x}$ & & & \\
\hline Intraventricular Hemorrnage & 0.621 & $0.038-10.236$ & 0.139 & & & \\
\hline Intraparenchymal Hemorrnage & 0.614 & $0.083-4.558$ & 0.634 & & & \\
\hline Concussion & 0.600 & $0.257-1.398$ & 0.237 & & & \\
\hline Major Fracture & 2.692 & $1.149-6.310$ & $0.023^{x}$ & & & \\
\hline
\end{tabular}

TABLE 4: Univariate and Multivariate Predictors of High-risk Medication Use at Discharge

BMI, body mass index; GC, Geriatric consultation; TBI, Traumatic brain injury; OR, Odds ratio; $\mathrm{Cl}$, Confidence interval

* Statistically significant $p<0.05$

\section{Discussion}

An early-implemented, proactive multidisciplinary trauma-geriatric model has consistently shown in the 
literature to prevent and successfully manage geriatric syndromes (i.e., delirium), preserve function, and facilitate discharge planning [12-13,18]. This study further supports this model by highlighting a significant difference (74\%) in high-risk medication use, specifically due to the lower utilization of benzodiazepines and sedatives upon discharge in the hospitalized elderly trauma patient. This difference was not seen in the GC and non-GC groups while hospitalized. One possibility for this findings is that the in-hospital high-risk medication use is a snapshot in time, i.e., the temporal pathway of high-risk medication use is unclear. Likely, the "at-discharge" time point reflects both a discontinuation and a reduction of high-risk medication use during the hospitalization and at the time of discharge.

Few studies have specifically assessed the impact of GC on high-risk medication prescriptions in the trauma population. However, there has been extensive research on high-risk medication use and its impact on falls and delirium [19]. The side effects of benzodiazepine use are well-established in both inpatient and outpatient settings in the geriatric population [7-10]. Brief interventions in the primary care setting have led to a significant reduction in benzodiazepine use in the elderly [20]. In the hospital, early proactive geriatric involvement in the care of an elderly trauma patient includes a comprehensive geriatric assessment (CGA). This multidimensional, multidisciplinary diagnostic instrument is designed to collect data on medical, psychosocial, and functional capabilities and the limitations of elderly patients, which aids in developing treatment and follow-up plans [2].

In this study, although the GC group had more frail patients (older, lower BMI, and slightly higher ISS), the readmission and LOS rates were similar in both groups. Moreover, these patients were more likely to go home (although this was not statistically significant). Many studies have looked at the positive impact of home discharge versus a skilled nursing facility (SNF) or an acute rehab facility on the medical, psychological, functional, and social state of elderly patients [21]. The positive impact of home care on elderly health outcomes includes the prevention of unplanned hospitalizations, a reduction in the number of hospital days, improvement in cognitive health, increased functional abilities, and improvements in quality of life [22-24]. This research suggests that a multidisciplinary trauma team that includes a geriatrician can increase the likelihood that elderly patients will be discharged home rather than to an SNF or acute rehab facility, which has positive implications for the medical and social health of these patients.

A large portion of the study population had a diagnosis of delirium prior to GC, limiting the examination of how GC affected this important syndrome. Consistent with prior literature, this study demonstrated that hyperactive delirium was more prevalent (i.e., documentation indicating the use of restraints/mitts, sitter, confusion/agitation, CAM scores, etc.) and more often diagnosed as well as treated than its counterpart, hypoactive delirium, in patients who received a GC [25]. The treatment of hypoactive and hyperactive delirium varies significantly. Diagnosing hypoactive delirium in older patients is key to ensure the apt execution of acute nonpharmacological treatment strategies such as reorientation and behavioral intervention [20]. Additionally, the timely diagnosis of hyperactive delirium is important so that appropriate pharmacological therapy can be administered to preserve patient safety [20]. In hyperactive delirium, inappropriate high-risk medication use, such as benzodiazepines, can worsen the condition and ultimately lead to increased mortality [2]. The long-term effects of delirium have been well-documented in the literature and include an increased risk of institutionalization, cognitive, and functional decline as well as mortality, especially with intensive care unit admission [26-28]. The etiology of delirium is multifactorial, involving genetics, direct brain insults (i.e. metabolic, inflammatory, and/or neurotransmitter abnormalities), and an aberrant stress response $[25,29]$. All three types, hyperactive, hypoactive, and mixed, of delirium can occur during a single hospitalization. The complex pathogenesis of delirium requires a multidisciplinary approach with the geriatrician for prevention, early detection, and management, especially in older patients.

A trauma-geriatric collaborative effort can change the culture of prescribing high-risk medications in the inpatient environment among a vulnerable population. In our institution, the standard of care for geriatric trauma patients has evolved to include the following: using low doses of high potency medications, attempting to prohibit benzodiazepine use unless clinically warranted, ordering standing intravenous or bymouth acetaminophen, frequent reorientations, slow weaning of standing pre-hospitalization high-risk medications (i.e. benzodiazepines), and active family involvement. Since this retrospective study, efforts have increased at our institution to enforce and standardize the criteria for a GC request (Figure 1). Elderly trauma patients with delirium on admission or the onset of delirium during hospitalization should also prompt a GC, a criterion that will likely need to be added to our protocol (Figure 1, Number 2). Understandably, the latter will aid with delirium management, but not prevention. Moreover, the timing of GC has been also enforced at HUMC to within 48 hours of patient admission since this study showed that most elderly patients were already delirious at the time of consultation. Furthermore, Figure 2 highlights the projected seamless flow of care of an elderly trauma patient from the emergency room (ER) to discharge. The geriatric trauma population is unique and requires constant vigilance, starting in the ER by flagging the electronic medical record (EMR) and the early involvement of the multidisciplinary team for rapid recovery and discharge. 


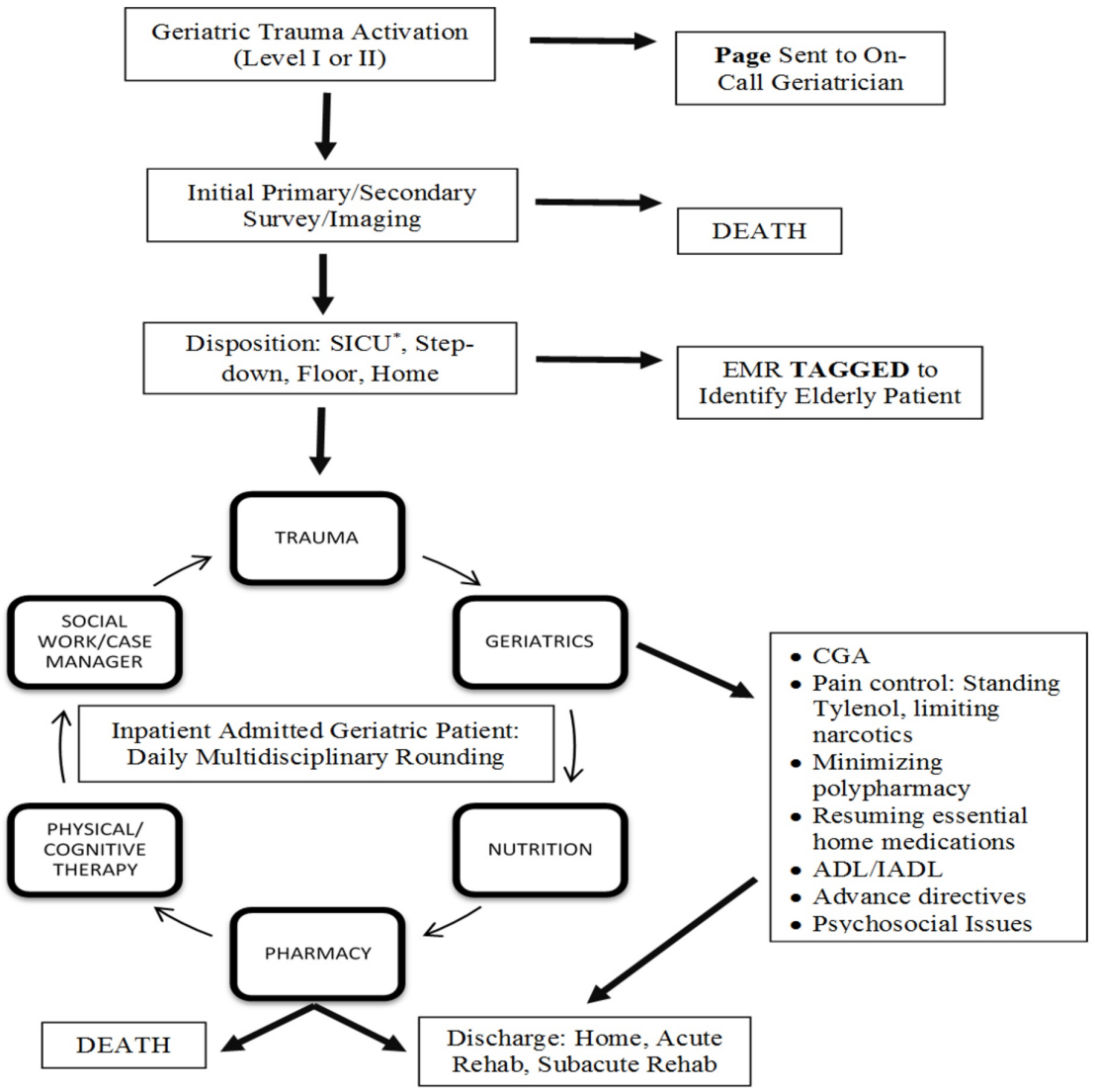

\section{FIGURE 2: Algorithm for a Multidisciplinary Team Approach to a Hospitalized Geriatric Trauma Patient}

SICU, Surgical critical care unit; EMR, Electronic medical record; CGA, Comprehensive geriatric assessment; $A D L$, Activities of daily living; IADL, Instrumental activities of daily living

There are several limitations to this study. First, this was a single-center retrospective study with a relatively small sample size and included only geriatric trauma patients. Thus, these results cannot be generalized to other populations or trauma centers. Second, this research was unable to determine if GC affected delirium incidence since a significant number of patients were already delirious prior to consultation. The selection bias of obtaining a GC likely affects the result of this study, i.e., most trauma surgeons obtained a GC in high-risk elderly patients (those with signs and symptoms of delirium). Furthermore, in cases where geriatricians are involved, there is likely actual heightened awareness of the primary providers that may be contributing to the overall decrease in the number of high-risk medications during the hospital course or at discharge. Additionally, this study did not track non-pharmacological interventions (i.e. physical therapy, family presence, hospital elder life program) for delirium [30]. This type of intervention may have contributed to the overall decrease in high-risk medication use at discharge in the GC group. Also, the reliability of the retrospective data extracted from the EMR is limited due to the possibility of poor clinical documentation. Lastly, there is no post-hospital follow-up to confirm that the discontinuation and/or avoidance of high-risk medications was persistent over a long period of time. There is also no data collected about post-hospital functional and cognitive outcomes. Further data will be needed to establish whether these changes are lasting and concomitant with behavioral changes in prescribing the high-risk medications.

Future studies should examine the impact of GC on-discharge medication reconciliation and post-discharge outcomes. The gold standard continues to be a prospective assessment of delirium and high-risk drug prescription use in the geriatric trauma population. Furthermore, extending this collaborative model to other services, such as neurosurgery, orthopedics, and general surgery, may reduce inappropriate high-risk medication use in the elderly.

\section{Conclusions}

As the population ages, a larger number of older patients are requiring hospital care due to traumatic injury. These patients are at a higher risk of delirium, leading to an increase in morbidity and mortality as compared to younger patients. This study further identifies that a GC reduces high-risk medication use upon discharge. A multidisciplinary trauma team, including a geriatrician, addresses the unique medical, psychological, functional, and social issues of this population. 


\section{Additional Information \\ Disclosures}

Human subjects: Consent was obtained by all participants in this study. Hackensack Meridian Health Institutional Review Board issued approval CR00003635. Animal subjects: All authors have confirmed that this study did not involve animal subjects or tissue. Conflicts of interest: In compliance with the ICMJE uniform disclosure form, all authors declare the following: Payment/services info: All authors have declared that no financial support was received from any organization for the submitted work. Financial relationships: All authors have declared that they have no financial relationships at present or within the previous three years with any organizations that might have an interest in the submitted work. Other relationships: All authors have declared that there are no other relationships or activities that could appear to have influenced the submitted work.

\section{References}

1. 2017 profile of older Americans. (2018). Accessed: November 27, 2018: https://www.acl.gov/sites/default/files/Aging and Disability in America/2017OlderAmericansProfile.pdf.

2. Committee on Trauma: ACS TQIP Geriatric Trauma Management guidelines . (2016). Accessed: November 27, 2018: https://www.facs.org/ /media/files/quality\%20programs/trauma/tqip/geriatric\%20guide\%20tqip.ashx.

3. 10 leading causes of death by age group, United States - 2013 . (2013). Accessed: November 27, 2018 : https://www.cdc.gov/injury/images/lc-charts/leading_causes_of_death_by_age_group_2013-a.gif.

4. Important facts about falls $\mid$ Home and recreational safety | CDC Injury Center . (2016). Accessed: November 27, 2018: https://www.cdc.gov/HomeandRecreationalSafety/Falls/adultfalls.html.

5. Bradburn E, Rodgers FB, Krasne M, et al.: High-risk geriatric protocol: improving mortality in the elderly . J Trauma Acute Care Surg. 2012, 73:435-440. 10.1097/TA.0b013e31825c7cf4

6. Leipzig RM, Curnrning RG, Tinetti ME: Drugs and falls in older people: a systematic review and metaanalysis: I psychotropic drugs. J Am Geriatr Soc. 1999, 47:30-39. 10.1111/j.1532-5415.1999.tb01898.x

7. Buffett-Jerrott SE, Stewart SH: Cognitive and sedative effects of benzodiazepine use . Curr Pharm Des. 2002, 8:45-58.

8. Hill KD, Wee R: Psychotropic drug-induced falls in older people: a review of interventions aimed at reducing the problem. Drugs \& Aging. 2012, 29:15-30. 10.2165/11598420-000000000-00000

9. Cumming RG, Le Couteur DG: Benzodiazepines and risk of hip fractures in older people: a review of the evidence. CNS Drugs. 2003, 17:825-837.

10. Meuleners LB, Duke J, Lee AH, Palamara P, Hildebrand J, Ng JQ: Psychoactive medications and crash involvement requiring hospitalization for older drivers: a population-based study. J Am Geriatr Soc. 2011, 59:1575-1580. 10.1111/j.1532-5415.2011.03561.x

11. Gurwitz JH, Field TS, Avorn J, et al.: Incidence and preventability of adverse drug events in nursing homes . Am J Med. 2000, 109:87-94.

12. Lenartowicz M, Parkovnick M, Mcfarlan A, et al.: An evaluation of a proactive geriatric trauma consultation service. Ann Surg. 2012, 256:1098-1101. 10.1097/SLA.0b013e318270f27a

13. Ellis G, Whitehead MA, O’Neill D, Langhorne P, Robinson D: Comprehensive geriatric assessment for older adults admitted to hospital. Cochrane Database Syst Rev. 2011, CD006211. 10.1002/14651858.CD006211.pub2

14. Fallon WF, Rader E, Zyzanski S, et al.: Geriatric outcomes are improved by a geriatric trauma consultation service. J Trauma Inj Infect Crit Care. 2006, 61:1040-1046. 10.1097/01.ta.0000238652.48008.59

15. American Psychiatric Association: Diagnostic and Statistical Manual of Mental Disorders [Internet]. 5th ed . American Psychiatric Association, Arlington, VA; 2013.

16. Inouye SK, van Dyck CH, Alessi CA, Balki S, Siegal AP, Horwitz RI: Clarifying confusion: the confusion assessment method. A new method for detection of delirium. Ann Intern Med. 1990, 113:941-948.

17. American Geriatrics Society 2015 Beers Criteria Update Expert Panel: American Geriatrics Society 2015. Updated Beers criteria for potentially inappropriate medication use in older adults. J Am Geriatr Soc. 2015, 63:2227-2246. 10.1111/jgs.13702

18. Mangram AJ, Mitchell CD, Shifflette VK, et al.: Geriatric trauma service: a one-year experience . J Trauma Acute Care Surg. 2012, 72:119-122. 10.1097/TA.0b013e318241foba

19. Woolcott JC, Richardson KJ, Wiens MO, et al.: Meta-analysis of the Impact of 9 medication classes on falls in elderly persons. Arch Intern Med. 2009, 169:1952-1960. 10.1001/archinternmed.2009.357

20. Mugunthan K, Mcguire T, Glasziou P: Minimal interventions to decrease long-term use of benzodiazepines in primary care: a systematic review and meta-analysis. Br J Gen Pract. 2011, 61:573-578. 10.3399/bjgp11X593857

21. Wiles JL, Leibing A, Guberman N, Reeve J, Allen RES: The meaning of "aging in place" to older people [Internet]. Gerontologist. 2012, 52:357-366. 10.1093/geront/gnr098

22. Neff DF, Madigan E, Narsavage G: APN-directed transitional home care model: achieving positive outcomes for patients with COPD. Home Healthc Nurse. 2003, 21:543-550.

23. Hughes SL, Ulasevich A, Weaver FM, Henderson W, Manheim L, Kubal JD, Bonarigo F: Impact of home care on hospital days: a meta analysis. Health Serv Res. 1997, 32:415-432.

24. Ellenbecker CH, Samia L, Cushman MJ, Alster K: Patient safety and quality in home health care . Healthcare Research and Quality, 2008.

25. MacLullich AMJ, Ferguson KJ, Miller T, de Rooij SE, Cunningham C: Unravelling the pathophysiology of delirium: a focus on the role of aberrant stress responses. J Psychosom Res. 2008, 65:229-238.

26. Witlox J, Eurelings L, de Jonghe J, et al.: Delirium in elderly patients and the risk of postdischarge mortality, institutionalization, and dementia: a meta-analysis. JAMA. 2010, 304:443-451. 10.1001/jama.2010.1013

27. Brummel NE, Jackson JC, Pandharipande PP, et al.: Delirium in the ICU and subsequent long-term disability 


\section{Cureus}

among survivors of mechanical ventilation. Crit Care Med. 2014, 42:369-377.

10.1097/CCM.0b013e3182a645bd

28. Brooks SE, Peetz AB: Evidence-based care of geriatric trauma patients. Surg Clin North Am. 2017, 97:1157-

1174. 10.1016/j.suc.2017.06.006

29. Gunther ML, Morandi A, Ely EW: Pathophysiology of delirium in the intensive care unit. Crit Care Clin.

2008, 24:45-65. 10.1016/j.ccc.2007.10.002

30. Bannon L, McGaughey J, Clarke M, McAuley DF, Blackwood B: Impact of non-pharmacological interventions on prevention and treatment of delirium in critically ill patients: protocol for a systematic review of quantitative and qualitative research. Syst Rev. 2016, 5:1-9. 10.1186/s13643-016-0254-0 